\title{
Hall and induced magnetic field effects on convective flow of viscoelastic fluid within an inclined channel with periodic surface conditions
}

\author{
Jitendra Kumar Singh, S. Vishwanath \\ Department of Mathematics, Vijayanagra Sri Krishnadevarya University, Ballari-583105, Karnataka, \\ India
}

Received: 4 July 2020; Received in revised form: 7 September 2020; Accepted: 18 September 2020; Published online 27 September 2020

(C) Published at www.ijtf.org

\begin{abstract}
This paper is concerned with the analytical study of the convective flow of a viscoelastic electrically conducting fluid within an inclined channel bounding a porous regime with Hall and induced magnetic field effects. An external magnetic field of high intensity is applied in the direction normal to the inclined surface. The left inclined surface of the channel is considered to be non-magnetic while the right inclined surface is assumed to be magnetized. Suitable non-dimensional transformations are used to reduce the problem to a similar nondimensional problem. The resulting flow governing equations are solved analytically. The consequences of various flow influencing parameters to the flow variables are numerically computed and presented in graphical and tabular form. It is interesting to note that the growth in angle of inclination reduces the induced magnetic field in the left half of the channel while this effect is opposite in the vicinity of the right inclined surface due to magnetization of this surface.
\end{abstract}

Keywords: Convective flow; viscoelastic fluid; magnetized surface; Hall current; induced magnetic field.

\section{Introduction}

Magnetohydrodynamic (MHD) mixed convective flow through a porous or nonporous regime is an important topic in the field of thermal science due to its numerous applications such as heat exchangers, nuclear reactor cooling, aeronautics, anthology of solar energy and so on. The study of mixed convection becomes important when both the free and force convection occur simultaneously and both the convective forces are comparable in magnitude. Motivated from these facts, recently, Mishra and Singh [1], Rashidi et al. [2], Sajid et al. [3], Nejad et al. [4], Seth and Singh [5], Reddy et al. [6], Ahmad et al. [7], Singh et al. [8-10] and Opanuga et al. [11] presented the MHD mixed convection flow problems. A comprehensive literature survey on MHD mixed convective buoyancy driven flows reports Corresponding e-mail: s.jitendrak@yahoo.com (Jitendra Kumar Singh) 
Name of First Author et al.

International Journal of Thermofluid Science and Technology (2020), Volume 7, Issue 4, Paper No. 070402

that in the most of the investigations magnetic diffusivity is considered to be large and induced magnetic field is neglected. However, the magnetic diffusivity is small for perfectly conducting working fluids. In such a situation the investigation of induced magnetic effect on MHD flow is significant. Seth and Sarkar [12] examined the induced magnetic field effect on hydromagnetic natural convection flow of a chemically reacting fluid over a moving vertical plate with ramped wall temperature. Sarveshanand and Singh [13] investigated the consequences of induced magnetic field on a free convective channel flow. Thermal radiation, chemical reaction and induced magnetic field effects on MHD mixed convection flow over a vertical porous plate is analyzed by Sarma and Pandit [14]. Subsequently, Ojjela et al. [15] analyzed the consequences of thermophoresis and induced magnetic field on mixed convective Jeffrey fluid within two porous plates. Interplay of conducting and nonconducting walls on MHD natural convection flow in a vertical micro-channel with induced magnetic field is presented by Jha and Aina [16]. Some more significant investigation on the topic are due to the researchers Seth and Singh [5], Singh et al. [8,10], Kumar et al. [17], Kumar et al. [18], Das et al. [19], Hayat et al. [20] and Raza et al. [21]. In the hydromagnetic flows, if the intensity of the applied magnetic field is very high or fluid is highly ionized, Hall current induces due to drifting and collision of ions about magnetic field lines. This current plays a prominent role in characterization of flow behavior. Motivated from these facts, recently, Singh et al. [22], Dharmaiah et al. [23] and Nanda and Kumbhakar [24] examined the Hall effect on buoyancy driven MHD boundary layer flows. The simultaneous influence of Hall current and induced magnetic field is examined by Seth and Singh [5] and Singh et al. $[8,10]$.

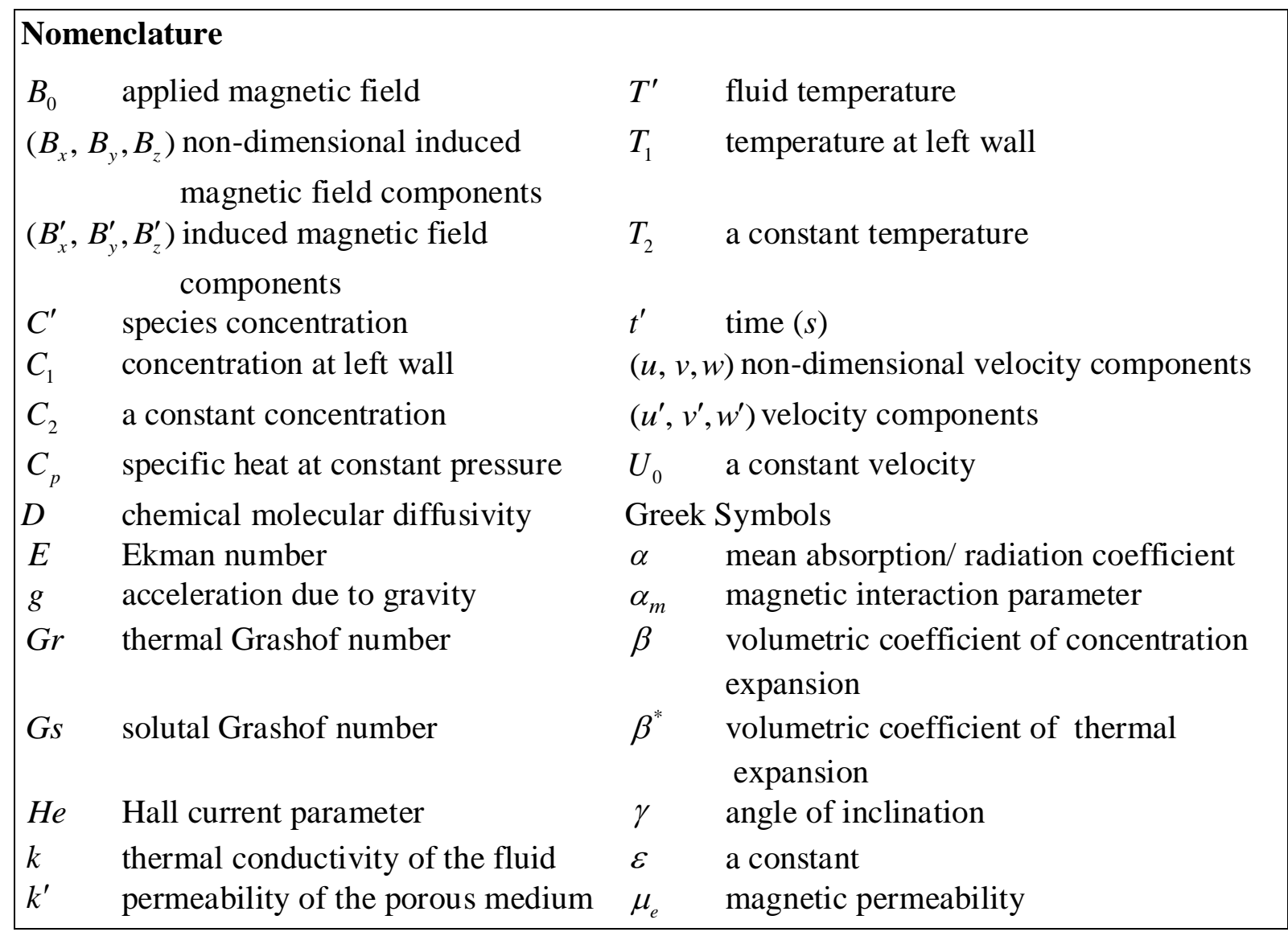


Name of First Author et al.

International Journal of Thermofluid Science and Technology (2020), Volume 7, Issue 4, Paper No. 070402

\begin{tabular}{|llll|}
\hline$k_{1}$ & permeability parameter & $v_{e}$ & kinematic viscosity \\
$k_{v}$ & viscoelastic parameter & $v_{m}$ & magnetic diffusivity \\
$k_{v}^{\prime}$ & viscoelastic constant & $\Omega$ & angular velocity \\
$p^{\prime}$ & pressure & $\omega$ & frequency parameter \\
$p$ & non-dimensional pressure & $\omega^{\prime}$ & frequency of oscillations \\
$P m$ & magnetic Prandtl number & $\phi$ & non-dimensional species concentration \\
$\operatorname{Pr}$ & Prandtl number & $\rho$ & fluid density \\
$R$ & a constant & $\sigma$ & electrical conductivity \\
$R^{\prime}$ & chemical reaction constant & $\theta$ & non-dimensional fluid temperature \\
$R c$ & chemical reaction parameter & $\tau$ & non-dimensional time \\
$S$ & heat source parameter & & \\
\hline
\end{tabular}

The investigation of viscoelastic fluid flow in the presence of magnetic field is a remarkably interesting subject due to its tremendous applications in biochemical and petrochemical industries. In practical point of view this subject is important due to production of complex multiphase products such as inks, paints and ceramic liquids. Stimulated from the diverse applications the eminent researchers [25-36] discussed the behaviour of viscoelastic fluid flow in the presence of magnetic field considering various flow configurations. In many industrial applications such as solar chimneys, ventilation devices and cooling systems where the heated surfaces are inclined, the angular location is important to study the convective heat transfer. Considering this fact into account Cimpean and Pop [37], Kumari and Radhakrishnamacharya [38], Srinivasacharya and Bindu [39], Hayat et al. [40], Mondal et al. [41], Ramesh Katta and Dewakar [42], Talukdar et al. [43], Garcia et al. [44], Raju et al. [45], Sharma et al. [46], Ngiangia and Jim-George [47], Noreen et al. [48], Etzold et al. [49] and Singh and Vishwanath [50] discussed the effect of angular location of the heated surface to the convective heat and mass transfer flows.

The purpose in this paper to examine the effects of Hall current and induced magnetic field together with the angular location of the heated surface on the MHD mixed convective flow of viscoelastic fluid within an inclined channel bounding porous regime. Further, in this paper we considered the left inclined channel surface to be non-magnetic while the right inclined surface is considered to be magnetized. The flow governing equations are solved analytically and solutions for fluid velocity, induced magnetic field, temperature and concentration are presented in closed form. It is noted that angle of inclination leads to decrement in the fluid velocity due to reduction of buoyancy forces. The induced magnetic field along the direction perpendicular to the main flow rises with angle of inclination.

\section{Formulation of the problem and solution}

In a rectangular Cartesian coordinate system, we considered the fully developed transient flow of an electrically conducting viscoelastic fluid within two inclined surfaces bounding a porous regime in the presence of a magnetic field of high intensity. The surfaces are inclined through an angle $\gamma$ with the vertical and the magnetic field $\vec{B}\left(0,0, B_{0}\right)$ is directed along the direction normal to the inclined surfaces. The flow system is influenced by the Coriolis force due to rigid body rotation of the flow system with angular velocity $\vec{\Omega}(0,0, \Omega)$ about the 
Name of First Author et al.

International Journal of Thermofluid Science and Technology (2020), Volume 7, Issue 4, Paper No. 070402

direction normal to the inclined surfaces. The left inclined non-magnetic surface is considered to be stationary and at constant temperature and concentration while the right inclined magnetized surface executes oscillations in its own plane and its temperature and concentration are fluctuating. The flow configuration of the physical problem is shown in the Fig. 1.

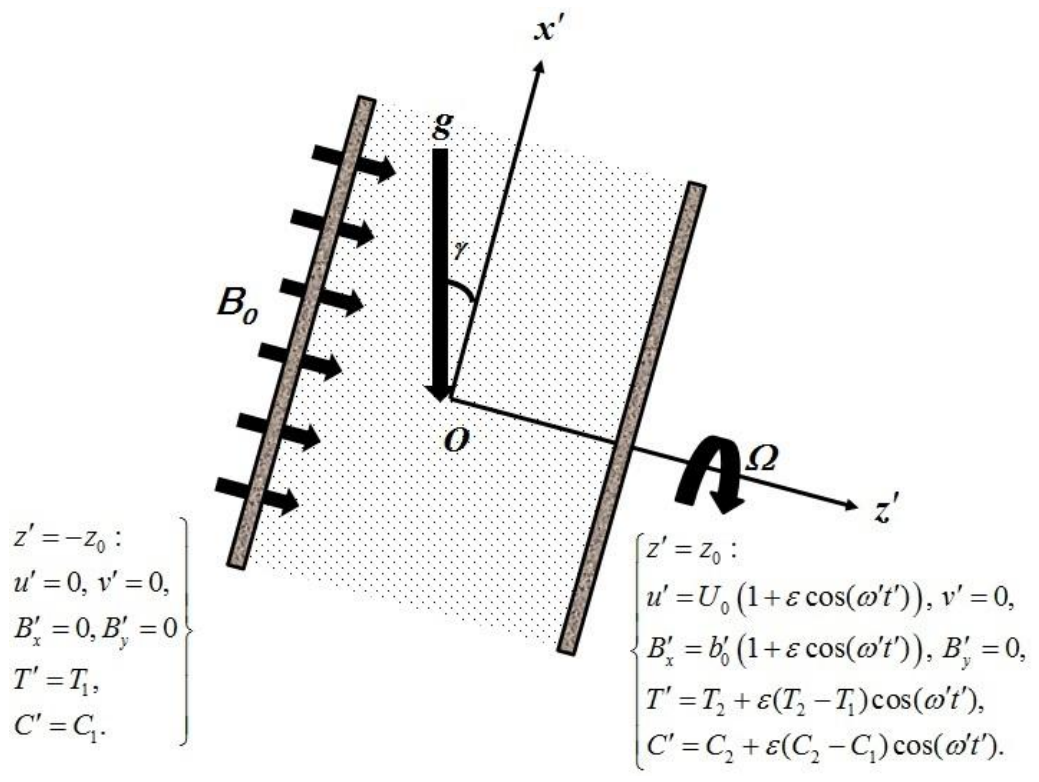

Fig. 1. Flow configuration of the physical problem

In accordance with these assumptions and Boussinesq approximations, the field equations governing fluid flow problem assume the following form:

the momentum equation:

$$
\begin{aligned}
& \frac{\partial u^{\prime}}{\partial t^{\prime}}-2 \Omega v^{\prime}=-\frac{1}{\rho} \frac{\partial p^{\prime}}{\partial x^{\prime}}+v_{e} \frac{\partial^{2} u^{\prime}}{\partial z^{\prime 2}}+\frac{B_{0}}{\rho \mu_{e}} \frac{\partial B_{x}^{\prime}}{\partial z^{\prime}}+\frac{k_{v}^{\prime}}{\rho} \frac{\partial^{3} u^{\prime}}{\partial z^{\prime 2} \partial t^{\prime}}-\frac{v_{e} u^{\prime}}{k^{\prime}}+g \beta\left(T^{\prime}-T_{1}\right) \cos \gamma \\
& +g \beta^{*}\left(C^{\prime}-C_{1}\right) \cos \gamma, \\
& \frac{\partial v^{\prime}}{\partial t^{\prime}}+2 \Omega u^{\prime}=v_{e} \frac{\partial^{2} v^{\prime}}{\partial z^{\prime 2}}+\frac{B_{0}}{\rho \mu_{e}} \frac{\partial B_{y}^{\prime}}{\partial z^{\prime}}+\frac{k_{v}^{\prime}}{\rho} \frac{\partial^{3} v^{\prime}}{\partial z^{\prime 2} \partial t^{\prime}}-\frac{v_{e} v^{\prime}}{k^{\prime}},
\end{aligned}
$$

the energy equation with heat source:

$$
\frac{\partial T^{\prime}}{\partial t^{\prime}}=\frac{k}{\rho C_{p}} \frac{\partial^{2} T^{\prime}}{\partial z^{\prime 2}}-\frac{4 \alpha^{2}\left(T^{\prime}-T_{1}\right)}{\rho C_{p}},
$$

the concentration equation with first order chemical reaction:

$$
\frac{\partial C^{\prime}}{\partial t^{\prime}}=D \frac{\partial^{2} C^{\prime}}{\partial z^{\prime 2}}-R^{\prime}\left(C^{\prime}-C_{1}\right) .
$$

In many industrial applications, in which the strength of applied magnetic field is strong or in the laboratory experiments where the fluid is highly ionized, there exists a current due to drifting and collision of ionized fluid particles about magnetic field lines. This current is called Hall current and this phenomenon known as Hall effect. In similar situations, 
Name of First Author et al.

International Journal of Thermofluid Science and Technology (2020), Volume 7, Issue 4, Paper No. 070402

the Hall current must be included in the generalized ohm's law. The magnetic induction equation with Hall effect is expressed as (Seth and Singh [5], Singh et al. [8, 10])

$$
\begin{aligned}
& \frac{\partial B_{x}^{\prime}}{\partial t^{\prime}}=B_{0} \frac{\partial u^{\prime}}{\partial z^{\prime}}+v_{m} \frac{\partial^{2} B_{x}^{\prime}}{\partial z^{\prime 2}}+v_{m} H e \frac{\partial^{2} B_{y}^{\prime}}{\partial z^{\prime 2}}, \\
& \frac{\partial B_{y}^{\prime}}{\partial t^{\prime}}=B_{0} \frac{\partial v^{\prime}}{\partial z^{\prime}}+v_{m} \frac{\partial^{2} B_{y}^{\prime}}{\partial z^{\prime 2}}-v_{m} H e \frac{\partial^{2} B_{x}^{\prime}}{\partial z^{\prime 2}} .
\end{aligned}
$$

The flow conditions at the inclined surfaces are:

At $z^{\prime}=-z_{0}: u^{\prime}=v^{\prime}=0, B_{x}^{\prime}=B_{y}^{\prime}=0, T^{\prime}=T_{1}, C^{\prime}=C_{1}$.

At $z^{\prime}=z_{0}:\left\{\begin{array}{l}u^{\prime}=U_{0}\left(1+\varepsilon \cos \left(\omega^{\prime} t^{\prime}\right)\right), v^{\prime}=0, B_{x}^{\prime}=b_{0}^{\prime}\left(1+\varepsilon \cos \left(\omega^{\prime} t^{\prime}\right)\right), B_{y}^{\prime}=0, \\ T^{\prime}=T_{2}+\left(T_{2}-T_{1}\right) \varepsilon \cos \left(\omega^{\prime} t^{\prime}\right), C^{\prime}=C_{2}+\left(C_{2}-C_{1}\right) \varepsilon \cos \left(\omega^{\prime} t^{\prime}\right) .\end{array}\right\}$

We introduce the following transformations to reduce the problem to a similar nondimensional problem

$$
\left.\begin{array}{l}
x=x^{\prime} / z_{0}, z=z^{\prime} / z_{0}, u=u^{\prime} / U_{0}, v=v^{\prime} / U_{0}, \tau=\Omega t^{\prime}, \omega=\omega^{\prime} / \Omega, \\
p=p^{\prime} / \rho U_{0} \Omega z_{0}, B_{x}=B_{x}^{\prime}(\Omega / v)^{1 / 2} / \sigma \mu_{e} U_{0} B_{0}, B_{y}=B_{y}^{\prime}(\Omega / v)^{1 / 2} / \sigma \mu_{e} U_{0} B_{0}, \\
\theta=\left(T^{\prime}-T_{1}\right) /\left(T_{2}-T_{1}\right), \phi=\left(C^{\prime}-C_{1}\right) /\left(C_{2}-C_{1}\right), b_{0}=b_{0}^{\prime}(\Omega / v)^{1 / 2} / \sigma \mu_{e} U_{0} B_{0}, \\
q=u+i v, B=B_{x}+i B_{y} .
\end{array}\right\}
$$

Use of above defined transformations reduce the equations (1)-(6) into following nondimensional form

$$
\begin{aligned}
& \frac{\partial q}{\partial \tau}+2 i q=-\frac{\partial p}{\partial x}+E \frac{\partial^{2} q}{\partial z^{2}}+2 \alpha_{m}^{2} E^{1 / 2} \frac{\partial B}{\partial z}-\frac{E q}{k_{1}}+k_{v} \frac{\partial^{3} q}{\partial z^{2} \partial t}+E G r \theta \cos \gamma+E G s \phi \cos \gamma, \\
& \operatorname{Pm} \frac{\partial B}{\partial \tau}=E^{1 / 2} \frac{\partial q}{\partial z}+E(1-i H e) \frac{\partial^{2} B}{\partial z^{2}}, \\
& \frac{\operatorname{Pr}}{E} \frac{\partial \theta}{\partial \tau}=\frac{\partial^{2} \theta}{\partial z^{2}}-S \theta, \\
& \frac{S c}{E} \frac{\partial \phi}{\partial \tau}=\frac{\partial^{2} \phi}{\partial z^{2}}-R c \phi,
\end{aligned}
$$

where

$$
\left.\begin{array}{l}
E=v_{e} / \Omega z_{0}^{2}, \alpha_{m}=(\sigma / 2 \rho \Omega)^{1 / 2} B_{0}, k_{1}=k^{\prime} / z_{0}^{2}, k_{v}=k_{v}^{\prime} / \rho z_{0}^{2}, \\
G r=g \beta z_{0}^{2}\left(T_{2}-T_{1}\right) / v U_{0}, G s=g \beta^{*} z_{0}^{2}\left(C_{2}-C_{1}\right) / v U_{0}, P m=v_{e} / v_{m}, \\
\operatorname{Pr}=v_{e} \rho C_{p} / k, S=4 \alpha^{2} z_{0}^{2} / k, S c=v_{e} / D \text { and } R c=R^{\prime} z_{0}^{2} / D .
\end{array}\right\}
$$

The associated boundary conditions in non-dimensional form are:

$$
\left.\begin{array}{ll}
q=B=\theta=\phi=0 & \text { at } \quad z=-1, \\
q=\theta=\phi=1+\varepsilon \cos (\omega \tau), \quad B=b_{0}(1+\varepsilon \cos (\omega \tau)) & \text { at } \quad z=1 .
\end{array}\right\}
$$


Name of First Author et al.

International Journal of Thermofluid Science and Technology (2020), Volume 7, Issue 4, Paper No. 070402

In order to find the solution of resulting equations (9)-(12) subject to the conditions (13), consider the fluid temperature, concentration, fluid velocity, induced magnetic field and pressure gradient along $x^{\prime}$-direction as

$$
\begin{aligned}
& \theta(z, \tau)=\theta_{0}(z)+\frac{\varepsilon}{2}\left(\theta_{1}(z) e^{i \omega \tau}+\theta_{2}(z) e^{-i \omega \tau}\right), \\
& \phi(z, \tau)=\phi_{0}(z)+\frac{\varepsilon}{2}\left(\phi_{1}(z) e^{i \omega \tau}+\phi_{2}(z) e^{-i \omega \tau}\right), \\
& q(z, \tau)=q_{0}(z)+\frac{\varepsilon}{2}\left(q_{1}(z) e^{i \omega \tau}+q_{2}(z) e^{-i \omega \tau}\right), \\
& B(z, \tau)=B_{0}(z)+\frac{\varepsilon}{2}\left(B_{1}(z) e^{i \omega \tau}+B_{2}(z) e^{-i \omega \tau}\right), \\
& \frac{\partial p}{\partial x}=R\left(1+\frac{\varepsilon}{2}\left(e^{i \omega \tau}+e^{-i \omega \tau}\right)\right) .
\end{aligned}
$$

On using equations (14)-(18) to the resulting flow governing partial differential equations, we obtain

$$
\begin{aligned}
& \theta_{0}^{\prime \prime}-S \theta_{0}=0, \\
& \phi_{0}^{\prime \prime}-R c \phi_{0}=0, \\
& E q_{0}^{\prime \prime}-\left(\left(E / k_{1}\right)+2 i\right) q_{0}+2 \alpha_{m}^{2} E^{1 / 2} B_{0}^{\prime}=-R-E G r \theta_{0} \cos \gamma-E G s \phi_{0} \cos \gamma, \\
& E(1-i H e) B_{0}^{\prime \prime}+E^{1 / 2} q_{0}^{\prime}=0, \\
& E \theta_{1}^{\prime \prime}-(S E+i \omega \operatorname{Pr}) \theta_{1}=0, \\
& E \phi_{1}^{\prime \prime}-(E R c+i \omega S c) \phi_{1}=0, \\
& \left(E+i \omega k_{v}\right) q_{1}^{\prime \prime}-\left(\left(E / k_{1}\right)+i(\omega+2)\right) q_{1}+2 \alpha_{m}^{2} E^{1 / 2} B_{1}^{\prime}=-R-E G r \theta_{1} \cos \gamma-E G s \phi_{1} \cos \gamma, \\
& E(1-i H e) B_{1}^{\prime \prime}+E^{1 / 2} q_{1}^{\prime}-i \omega P m B_{1}=0, \\
& E \theta_{2}^{\prime \prime}-(S E-i \omega \operatorname{Pr}) \theta_{2}=0, \\
& E \phi_{2}^{\prime \prime}-(E R c-i \omega S c) \phi_{2}=0, \\
& \left(E-i \omega k_{v}\right) q_{2}^{\prime \prime}-\left(\left(E / k_{1}\right)-i(\omega-2)\right) q_{2}+2 \alpha_{m}^{2} E^{1 / 2} B_{2}^{\prime}=-R-E G r \theta_{2} \cos \gamma-E G s \phi_{2} \cos \gamma, \\
& E(1-i H e) B_{2}^{\prime \prime}+E^{1 / 2} q_{2}^{\prime}+i \omega P m B_{2}=0 .
\end{aligned}
$$

Use of equations (14) to (18) to the boundary conditions (13), gives

$$
\left.\begin{array}{l}
\theta_{0}=\theta_{1}=\theta_{2}=0, \phi_{0}=\phi_{1}=\phi_{2}=0, q_{0}=q_{1}=q_{2}=0, B_{0}=B_{1}=B_{2}=0 \quad \text { at } z=-1, \\
\theta_{0}=\theta_{1}=\theta_{2}=1, \phi_{0}=\phi_{1}=\phi_{2}=1, q_{0}=q_{1}=q_{2}=1, B_{0}=B_{1}=B_{2}=b_{0}=1 \text { (assume) at } z=1 .
\end{array}\right\}
$$

The solutions of equations (19)-(30) subject to the boundary conditions (31) are expressed as 
Name of First Author et al.

International Journal of Thermofluid Science and Technology (2020), Volume 7, Issue 4, Paper No. 070402

$$
\begin{aligned}
& \theta_{i}=\frac{\sinh \left(r_{i}(1+z)\right)}{\sinh \left(2 r_{i}\right)}, \quad i=0,1,2 \\
& \phi_{i}=\frac{\sinh \left(s_{i}(1+z)\right)}{\sinh \left(2 s_{i}\right)}, \quad i=0,1,2 \\
& q_{0}=\frac{\sinh \left(a_{0}(1+z)\right)}{\sinh \left(2 a_{0}\right)}+\frac{\operatorname{Grcos} \gamma}{\left(r_{0}^{2}-a_{0}^{2}\right)}\left\{\frac{\sinh \left(a_{0}(1+z)\right)}{\sinh \left(2 a_{0}\right)}-\frac{\sinh \left(r_{0}(1+z)\right)}{\sinh \left(2 r_{0}\right)}\right\} \\
& +\frac{G \operatorname{scos} \gamma}{\left(s_{0}^{2}-a_{0}^{2}\right)}\left\{\frac{\sinh \left(a_{0}(1+z)\right)}{\sinh \left(2 a_{0}\right)}-\frac{\sinh \left(s_{0}(1+z)\right)}{\sinh \left(2 s_{0}\right)}\right\}+c_{01}\left(1-\frac{\cosh \left(a_{0} z\right)}{\cosh a_{0}}\right) \text {, } \\
& q_{i}=\frac{\sinh \left(a_{i 1}(1+z)\right)}{\sinh \left(2 a_{i 1}\right)}+c_{i 1}\left\{\cosh \left(a_{i 2} z\right)-\frac{\cosh \left(a_{i 1} z\right) \cosh \left(a_{i 2}\right)}{\cosh \left(a_{i 1}\right)}\right\} \\
& +c_{i 2}\left\{\sinh \left(a_{i 2} z\right)-\frac{\sinh \left(a_{i 1} z\right) \sinh \left(a_{i 2}\right)}{\sinh \left(a_{i 1}\right)}\right\}+\frac{z_{i 3} R}{E z_{i 2}}\left\{1-\frac{\cosh \left(a_{i 1} z\right)}{\cosh \left(a_{i 1}\right)}\right\} \\
& +\frac{\left(r_{i}^{2} z_{i 4}-z_{i 3}\right) G r \cos \gamma}{\left(r_{i}^{4}-z_{i 1} r_{i}^{2}+z_{i 2}\right)}\left\{\frac{\sinh \left(a_{i 1}(1+z)\right)}{\sinh \left(2 a_{i 1}\right)}-\frac{\sinh \left(r_{i}(1+z)\right)}{\sinh \left(2 r_{i}\right)}\right\} \\
& +\frac{\left(s_{i}^{2} z_{i 4}-z_{i 3}\right) G \cos \gamma}{\left(s_{i}^{4}-z_{i 1} s_{i}^{2}+z_{i 2}\right)}\left\{\frac{\sinh \left(a_{i 1}(1+z)\right)}{\sinh \left(2 a_{i 1}\right)}-\frac{\sinh \left(s_{i}(1+z)\right)}{\sinh \left(2 s_{i}\right)}\right\}, \quad i=1,2 \\
& B_{0}=\frac{1}{2 \alpha_{m}^{2} E^{1 / 2}}\left[E _ { 2 } \left\{\frac{\cosh \left(a_{0}(1+z)\right)}{a_{0} \sinh \left(2 a_{0}\right)}-\frac{c_{01} \sinh \left(a_{0} z\right)}{a_{0} \cosh a_{0}}\right.\right. \\
& +\frac{G r \cos \gamma}{\left(r_{0}^{2}-a_{0}^{2}\right)}\left(\frac{\cosh \left(a_{0}(1+z)\right)}{a_{0} \sinh \left(2 a_{0}\right)}-\frac{\cosh \left(r_{0}(1+z)\right)}{r_{0} \sinh \left(2 r_{0}\right)}\right) \\
& \left.\left.+\frac{G \operatorname{scos} \gamma}{\left(s_{0}^{2}-a_{0}^{2}\right)}\left(\frac{\cosh \left(a_{0}(1+z)\right)}{a_{0} \sinh \left(2 a_{0}\right)}-\frac{\cosh \left(s_{0}(1+z)\right)}{s_{0} \sinh \left(2 s_{0}\right)}\right)\right\}+z\left(c_{01} E_{1}-R\right)+c_{02}\right] \text {, } \\
& B_{i}= \pm \frac{1}{2 \alpha_{m}^{2} E^{1 / 2}}\left[\frac{a_{i 1}^{\prime} \cosh \left(a_{i 1}(1+z)\right)}{\sinh \left(2 a_{i 1}\right)}+c_{i 1}\left\{a_{i 2}^{\prime} \sinh \left(a_{i 2} z\right)-\frac{a_{i 1}^{\prime} \sinh \left(a_{i 1} z\right) \cosh \left(a_{i 2}\right)}{\cosh \left(a_{i 1}\right)}\right\}\right. \\
& +c_{i 1}\left\{a_{i 2}^{\prime} \cosh \left(a_{i 2} z\right)-\frac{a_{i 1}^{\prime} \cosh \left(a_{i 1} z\right) \sinh \left(a_{i 2}\right)}{\sinh \left(a_{i 1}\right)}\right\}-\frac{z_{i 3} R}{E z_{i 2}} \frac{a_{i 1}^{\prime} \sinh \left(a_{i 1} z\right)}{\cosh \left(a_{i 1}\right)} \\
& +\frac{\left(r_{i}^{2} z_{i 4}-z_{i 3}\right) G r \cos \gamma}{\left(r_{i}^{4}-z_{i 1} r_{i}^{2}+z_{i 2}\right)}\left\{\frac{a_{i 1}^{\prime} \cosh \left(a_{i 1}(1+z)\right)}{\sinh \left(2 a_{i 1}\right)}-\frac{r_{i 1}^{\prime} \cosh \left(r_{i}(1+z)\right)}{\sinh \left(2 r_{i}\right)}\right\} \\
& +\frac{\left(s_{i}^{2} z_{i 4}-z_{i 3}\right) G \operatorname{scos} \gamma}{\left(s_{i}^{4}-z_{i 1} s_{i}^{2}+z_{i 2}\right)}\left\{\frac{a_{i 1}^{\prime} \cosh \left(a_{i 1}(1+z)\right)}{\sinh \left(2 a_{i 1}\right)}-\frac{s_{i 1}^{\prime} \cosh \left(s_{i}(1+z)\right)}{\sinh \left(2 s_{i}\right)}\right\}, \quad i=1,2 .
\end{aligned}
$$

the $+v e$ and $-v e$ signs in the above equation are, respectively, for $B_{1}$ and $B_{2}$. 
Name of First Author et al.

International Journal of Thermofluid Science and Technology (2020), Volume 7, Issue 4, Paper No. 070402

Use of solutions (32)-(37) to the equations (14)-(17) give the desired fluid temperature, concentration, fluid velocity and induced magnetic field.

The skin friction coefficient at the non-magnetic and magnetized surfaces of the channel is obtained with the assistance of the following equations

$$
\left.\begin{array}{l}
\tau_{1}=\tau_{x 1}+i \tau_{y 1}=\left[q_{0}^{\prime}+(\varepsilon / 2)\left(q_{1}^{\prime} e^{i \omega \tau}+q_{2}^{\prime} e^{-i \omega \tau}\right)\right]_{z=-1}, \\
\tau_{2}=\tau_{x 2}+i \tau_{y 2}=\left[q_{0}^{\prime}+(\varepsilon / 2)\left(q_{1}^{\prime} e^{i \omega \tau}+q_{2}^{\prime} e^{-i \omega \tau}\right)\right]_{z=1} \cdot
\end{array}\right\}
$$

The heat and mass transfer rates at the non-magnetic and magnetized surfaces of the channel are expressed in terms of Nusselt and Sherwood numbers as

$$
\left.\left.\begin{array}{l}
N u_{1}=-\left[N u_{10}+(\varepsilon / 2)\left(N u_{11} e^{i \omega \tau}+N u_{12} e^{-i \omega \tau}\right)\right], \\
N u_{2}=-\left[N u_{20}+(\varepsilon / 2)\left(N u_{21} e^{i \omega \tau}+N u_{22} e^{-i \omega \tau}\right)\right],
\end{array}\right\} \begin{array}{l}
S h_{1}=-\left[S h_{10}+(\varepsilon / 2)\left(S h_{11} e^{i \omega \tau}+S h_{12} e^{-i \omega \tau}\right)\right], \\
S h_{2}=-\left[S h_{20}+(\varepsilon / 2)\left(S h_{21} e^{i \omega \tau}+S h_{22} e^{-i \omega \tau}\right)\right],
\end{array}\right\}
$$

where

$$
\left.\begin{array}{l}
N u_{1 i}=\frac{r_{i}}{\sinh \left(2 r_{i}\right)}, \quad N u_{2 i}=r_{i} \frac{\cosh \left(2 r_{i}\right)}{\sinh \left(2 r_{i}\right)}, \quad i=0,1,2 \\
S h_{1 i}=\frac{s_{i}}{\sinh \left(2 s_{i}\right)}, \quad S h_{2 i}=s_{i} \frac{\cosh \left(2 s_{i}\right)}{\sinh \left(2 s_{i}\right)} \cdot i=0,1,2
\end{array}\right\}
$$

\section{Results and discussion}

In this section, the analytical solutions for velocity field, induced magnetic field, temperature, concentration, skin friction coefficient, Nusselt and Sherwood numbers at both the surfaces are computed. The consequences of various flow parameters to these flow variables are presented in graphical and tabular forms. Figures 2-6 represents the velocity profiles whereas Figs. 7-11 demonstrate the induced magnetic profiles correspond to various system parameters. Figures 12 and 13 are, respectively; illustrate the temperature and concentration profiles. In the numerical computation the default values of system parameters are taken as $R=1, \varepsilon=1, \gamma=\pi / 6, H e=0.5, \alpha_{m}^{2}=5, P m=0.7, E=0.5, \omega \tau=\pi / 2, k_{v}=0.25$, $G r=4, G s=5, \operatorname{Pr}=0.71, S=2, S c=0.22$ and $R c=0.2$.

\subsection{Velocity}

Figure 2 illustrates that the angle of inclination leads to a decrement in the velocity. This is due to the fact that the increment in the angle of inclination causes reduction in the buoyancy effects which decelerate the fluid velocity. Hall effect on fluid flow is shown in Fig. 3. It can be easily noticed that Hall current has tendency to reduce main flow while it has growing tendency on the flow perpendicular to the main flow. This is due to the reason that 
Name of First Author et al.

International Journal of Thermofluid Science and Technology (2020), Volume 7, Issue 4, Paper No. 070402

the Hall current induces due to spiraling and collision of ions about magnetic field lines. The magnetic diffusion consequence on the flow behavior is plotted in Fig. 4. Rising values of magnetic diffusion leads to rise the main flow while reduce the flow perpendicular to the main flow. Magnetic diffusion brings decrement in the induced magnetic field along the main flow. Thus, on raising the magnetic diffusion, the magnetic drag force reduces along main flow. Hence flow velocity raises in this direction. Figure 5 illustrates the effect of Ekman number on the fluid flow. A reduction effect is observed on the velocity profiles by Ekman number. Ekman number measures the relative strength of viscous force to the Coriolis force. It falls down for the increasing values of the Coriolis force. The direction of Coriolis force is perpendicular to the main flow thus it has tendency to bring increment in the velocity profile perpendicular to the main flow. Due to this reason the velocity perpendicular to the main flow reduces with Ekman number. Figure 6 show that along the main flow, viscoelastic parameter has rising effect on the velocity while perpendicular to the main flow it has reducing tendency on the velocity.

\subsection{Induced magnetic field}

The induced magnetic field corresponding to various angle of inclination is exhibited in Fig. 7. It is seen that induced magnetic field along the main flow fall down on rising angle of inclination throughout the channel except in the vicinity of the magnetized surface. Angle of inclination leads to raise the induced magnetic field in the direction perpendicular to the main flow. Hall effect on induced magnetic field can be observed in the Fig. 8. Hall current has tendency to decrease induced magnetic field along the main flow because the direction of the Hall current is perpendicular to both the magnetic field and flow direction. The reverse effect is observed on the induced magnetic field perpendicular to the main flow direction in the neighborhood of the magnetized surface. Figure 9 indicates that magnetic diffusion leads to decrement in the induced magnetic field along the main flow due to the leakage of magnetic field. Its effect on the induced magnetic field perpendicular to the main flow is opposite in the region close to the magnetized surface. The behavior of Coriolis force on induced magnetic field is presented in Fig. 10. A growth is brought out by Coriolis force in the induced magnetic field along the main flow. It leads to decrement in the induced magnetic field perpendicular to the main flow in the left half of the channel. Figure 11 illustrates the impacts of the viscoelastic parameter on the induced magnetic field. It is noted that induced magnetic field along the main flow grows on growing viscoelastic parameter. Similar influence is observed on the induced magnetic field perpendicular to the main flow in the neighboring region of the magnetized surface.

\subsection{Temperature and concentration}

Figure 12 and 13 illustrate that Ekman number reduces fluid temperature and concentration i.e. this concludes that Coriolis force leads to grow on fluid temperature and concentration.

\subsection{Quantities of physical interest}

Table 1 demonstrates the skin friction coefficient at both the non-magnetic and magnetized surfaces. Angle of inclination has tendency to grow the skin friction coefficient along the main flow while it has reverse tendency on the skin friction coefficient perpendicular to the main flow. Hall effect shows in the opposite behavior as that of angle of 
Name of First Author et al.

International Journal of Thermofluid Science and Technology (2020), Volume 7, Issue 4, Paper No. 070402

inclination on the skin friction coefficient at both the non-magnetic and magnetized surfaces. This is because it has flow inducing tendency in the direction perpendicular to the main flow. Magnetic diffusion leads to decrease skin friction coefficient at both the surfaces along main flow and perpendicular to the main flow directions because magnetic diffusion has tendency to reduce magnetic drag force. Along the main flow rotation brings decrement in the skin friction coefficient at the non-magnetic surface while it brings increment in the skin friction at the magnetized surface. Along the direction perpendicular to the main flow rotation leads to a decrement in the skin friction coefficient at both the surfaces. This is due to the fact that Coriolis force has flow inducing tendency perpendicular to the main flow similar to the Hall current. Viscoelastic parameter show the opposite influence as that of Coriolis force on the skin friction coefficient along the main flow while it shows the similar influence as that of Coriolis force on the skin friction coefficient perpendicular to the main flow. It can be easily concluded from table 2 that rotation raises the heat and mass transfer rates at the nonmagnetic surface while it reduces these on the magnetized surface.

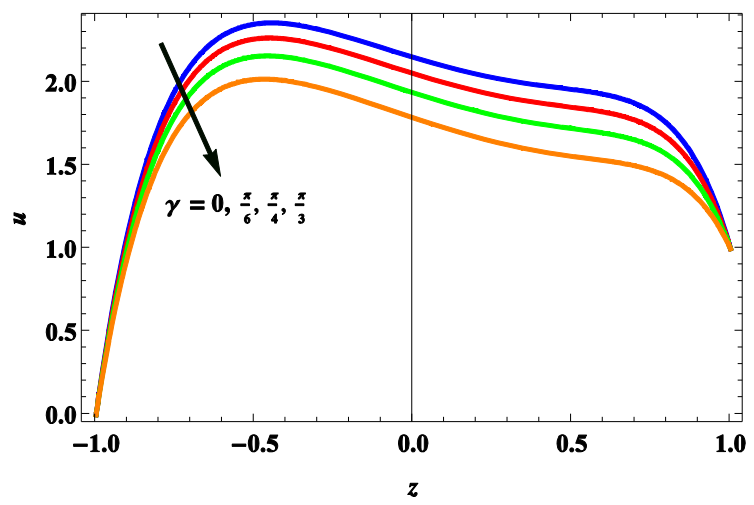

(a)

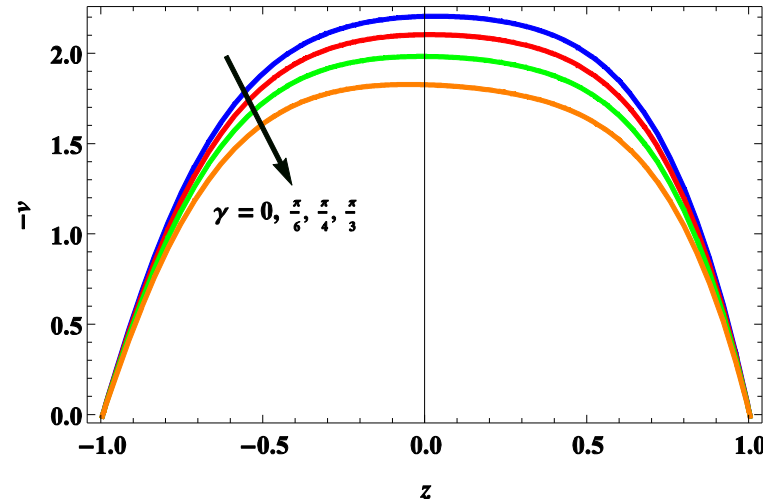

(b)

Fig. 2. Velocity profiles for $\gamma$ along the (a) main flow and (b) perpendicular to main flow directions.

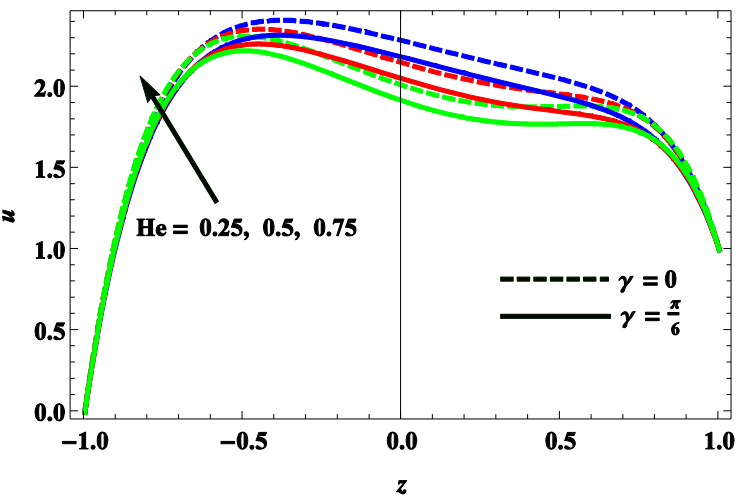

(a)

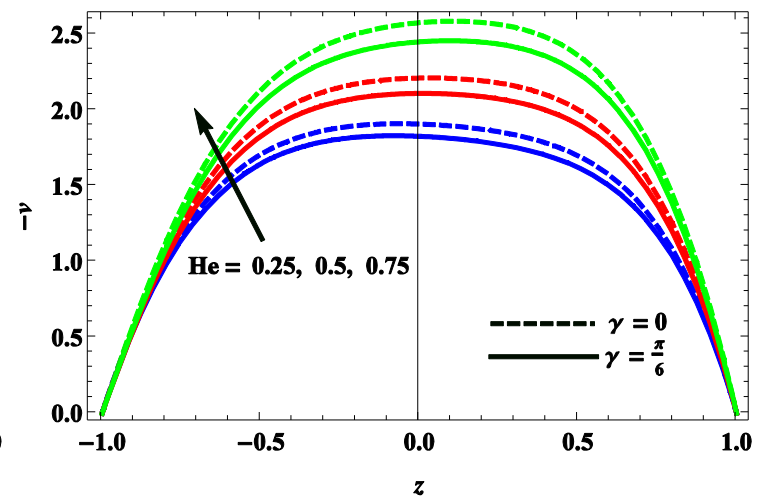

(b)

Fig. 3. Velocity profiles for $\mathrm{He}$ along the (a) main flow and (b) perpendicular to main flow directions. 
Name of First Author et al.

International Journal of Thermofluid Science and Technology (2020), Volume 7, Issue 4, Paper No. 070402

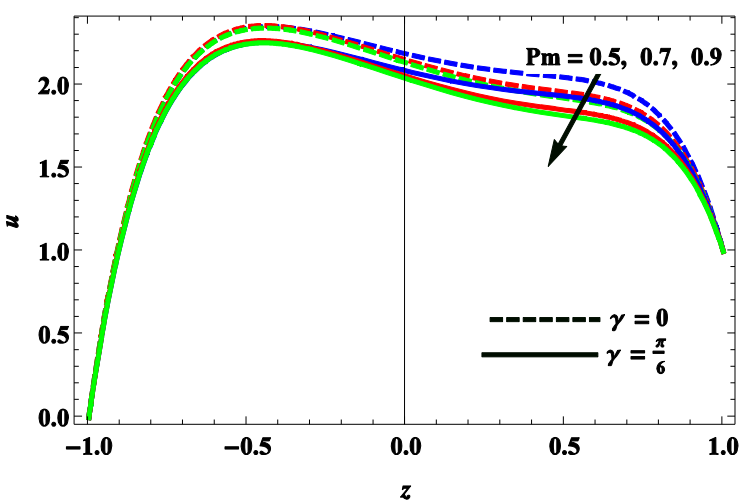

(a)

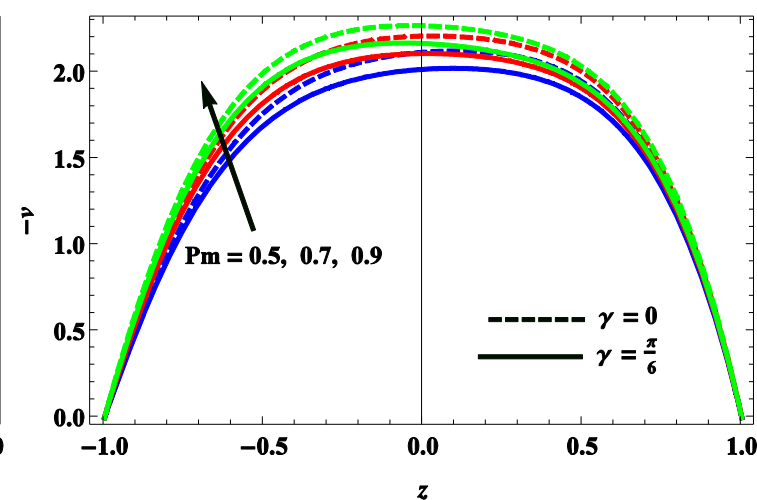

(b)

Fig. 4. Velocity profiles for $P m$ along the (a) main flow and (b) perpendicular to main flow directions.

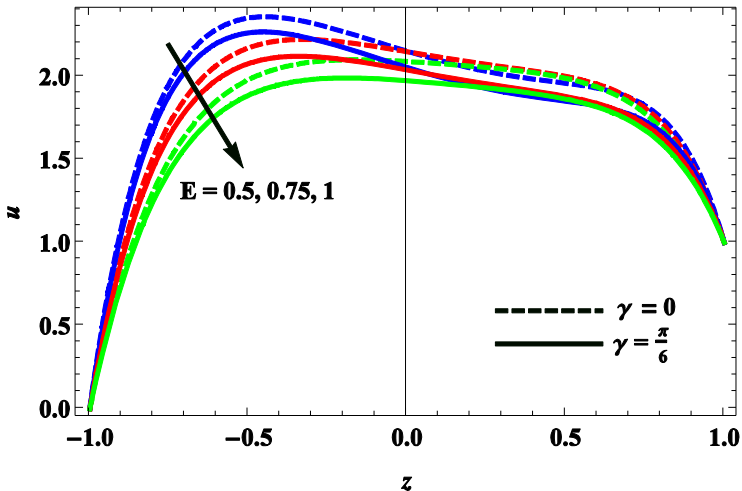

(a)

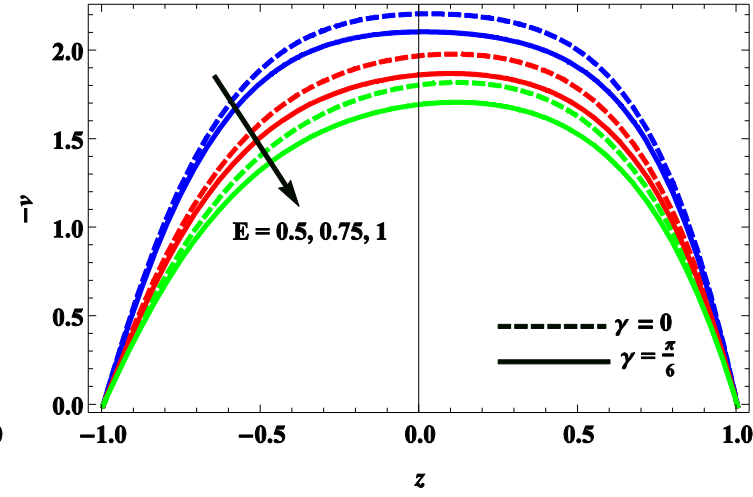

(b)

Fig. 5. Velocity profiles for $E$ along the (a) main flow and (b) perpendicular to the main flow directions.

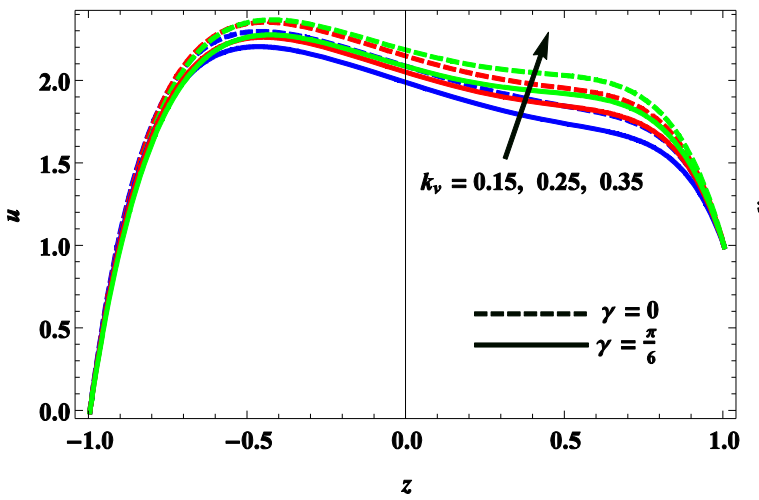

(a)

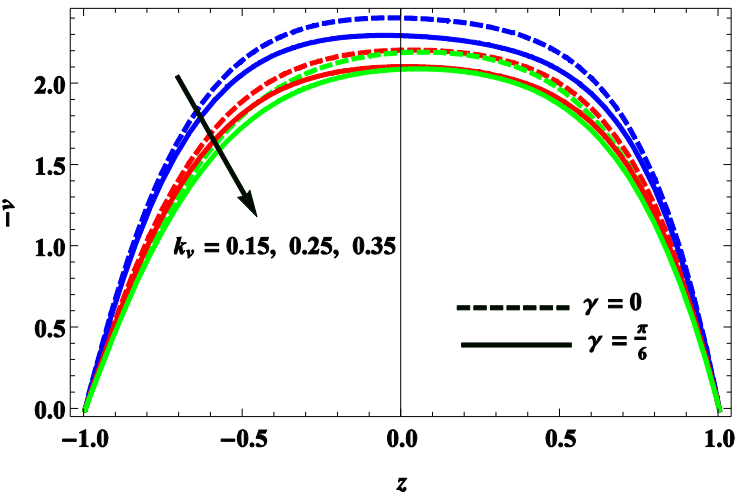

(b)

Fig. 6. Velocity profiles for $k_{v}$ along the (a) main flow and (b) perpendicular to the main flow directions. 


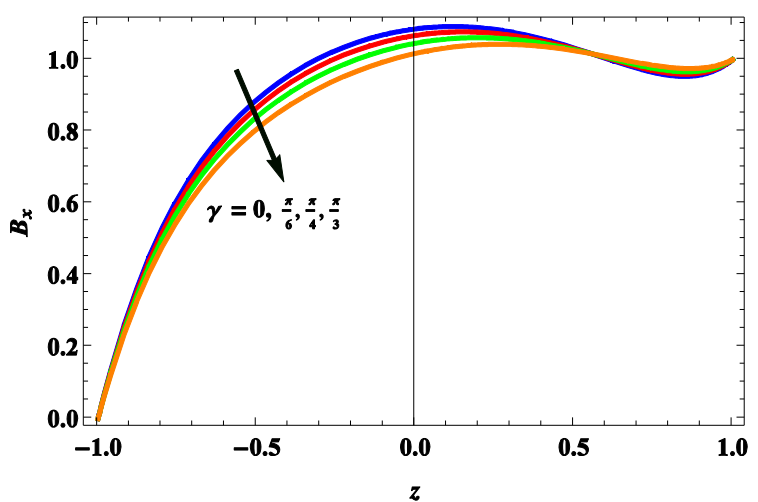

(a)

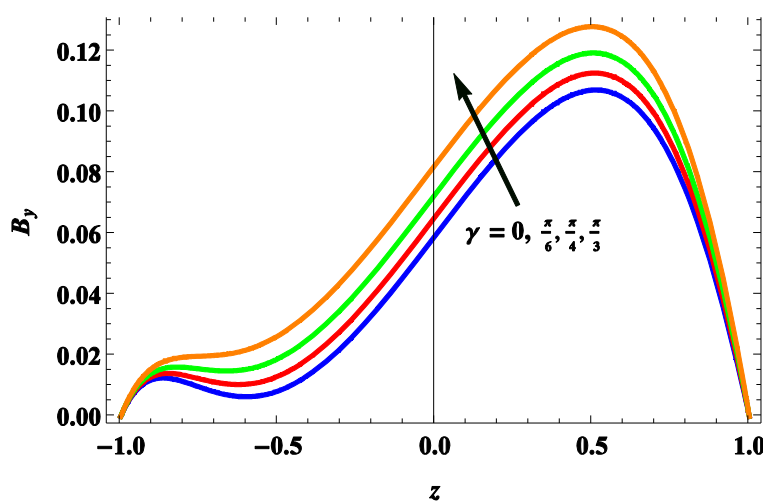

(b)

Fig. 7. Induced magnetic field profiles for $\gamma$ along (a) main flow and (b) perpendicular to the main flow directions.

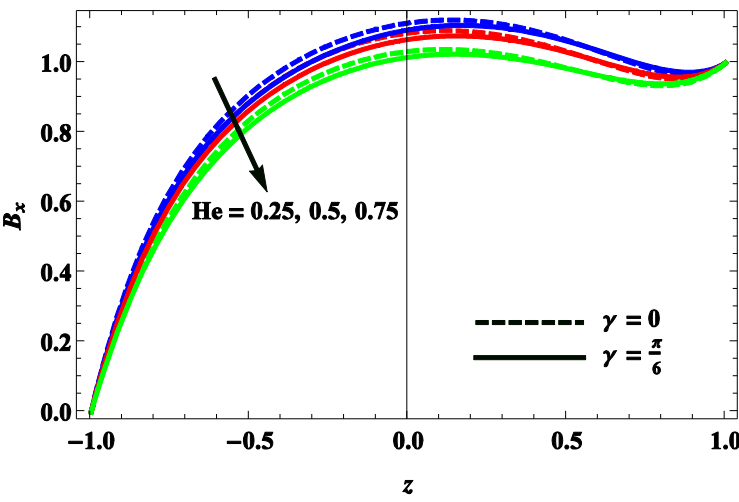

(a)

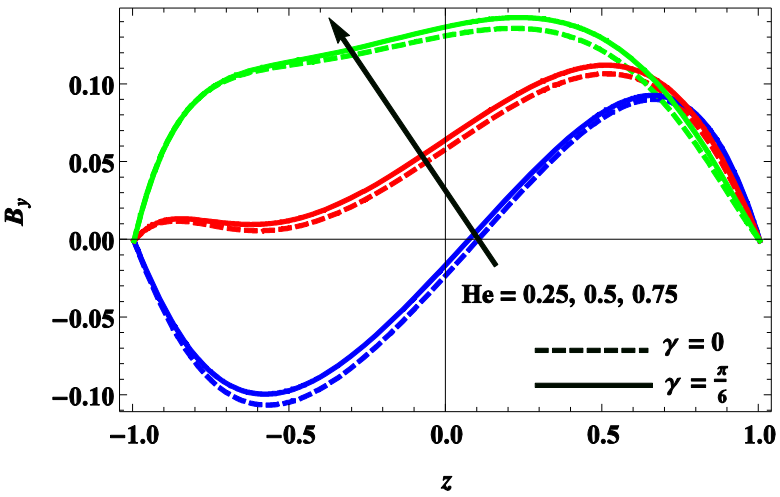

(b)

Fig. 8. Induced magnetic field profiles for $\mathrm{He}$ along the (a) main flow and (b) perpendicular to the main flow directions.

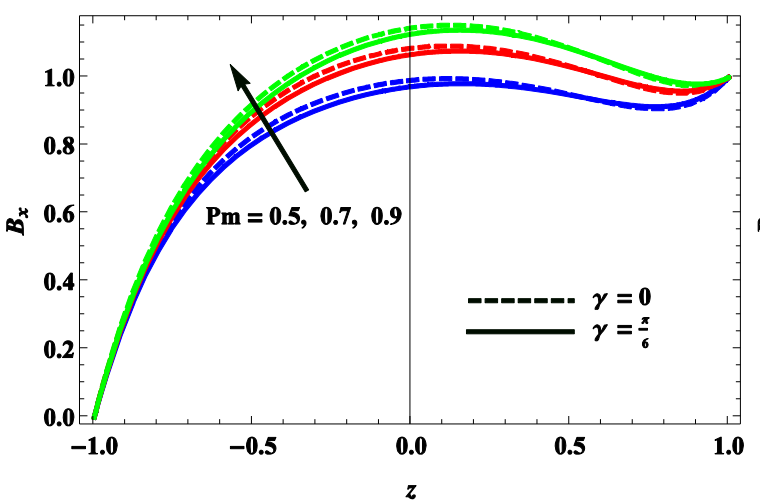

(a)

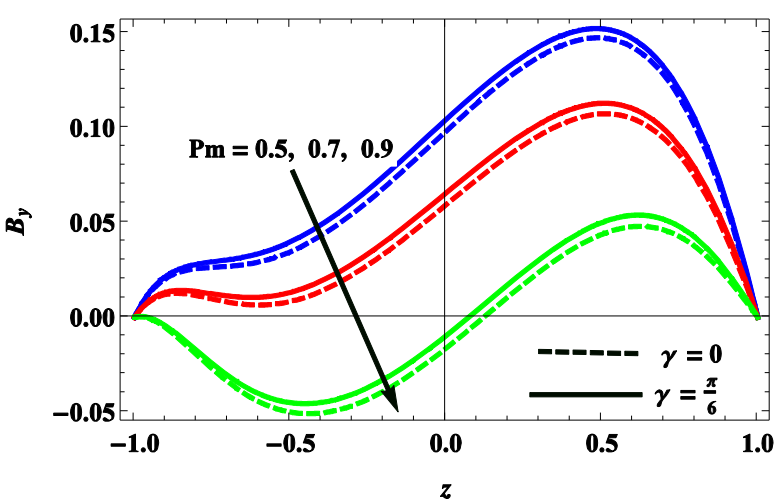

(b)

Fig. 9. Induced magnetic field profiles for $P m$ along the (a) main flow and (b) perpendicular to the main flow directions. 
Name of First Author et al.

International Journal of Thermofluid Science and Technology (2020), Volume 7, Issue 4, Paper No. 070402

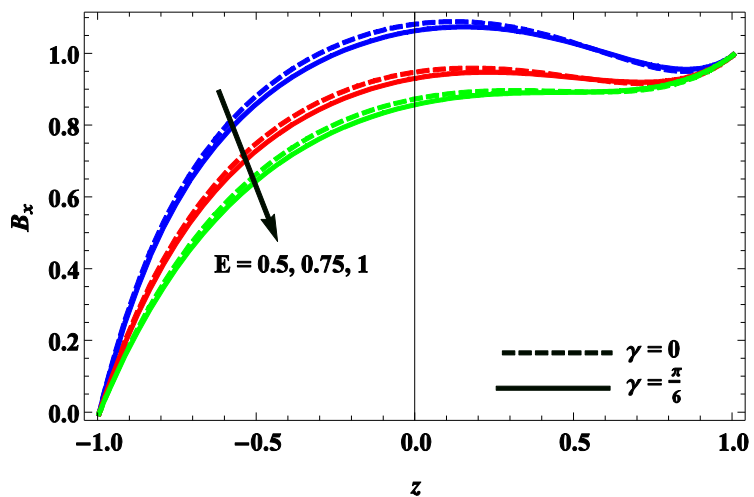

(a)

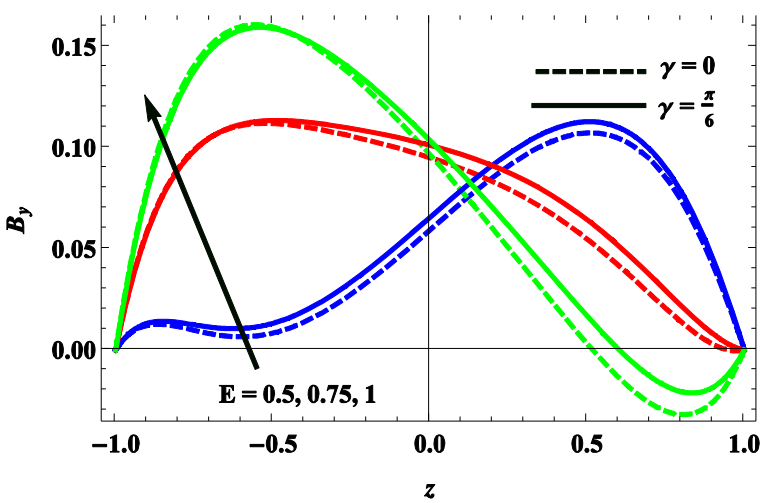

(b)

Fig. 10. Induced magnetic field profiles for $E$ along the (a) main flow and (b) perpendicular to the main flow directions.

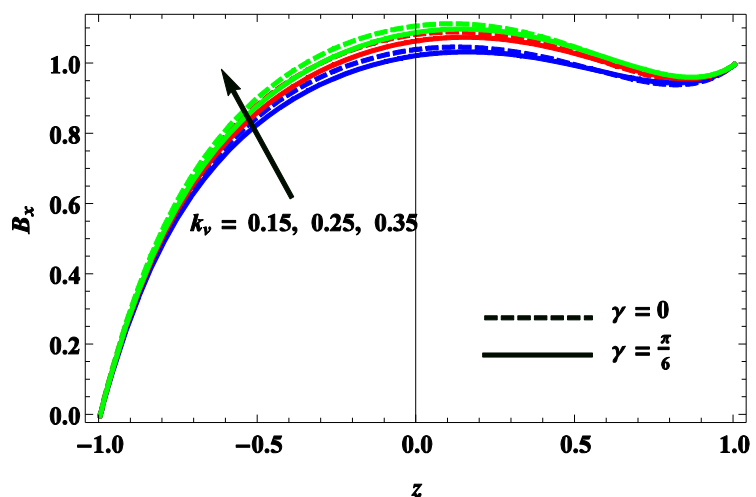

(a)

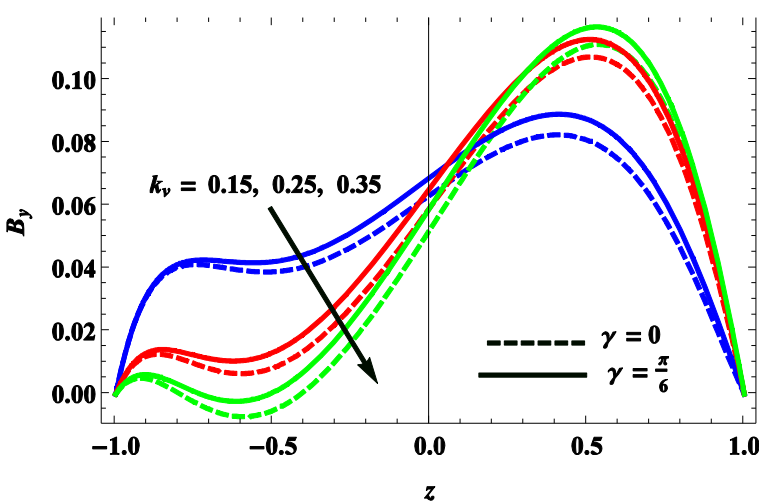

(b)

Fig. 11. Induced magnetic field profiles for $k_{v}$ along the (a) main flow (b) perpendicular to the main flow directions.

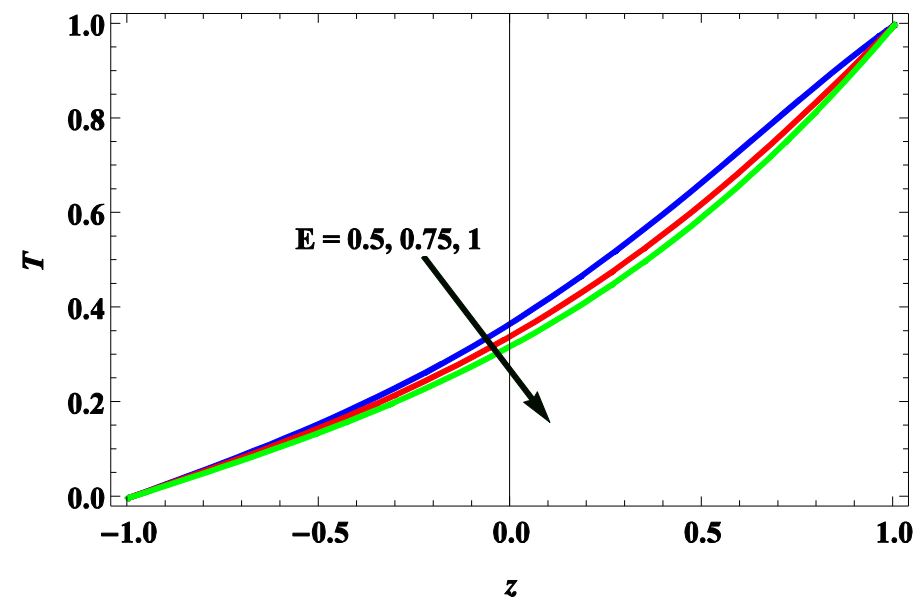

Fig. 12. Temperature profile for $E$. 


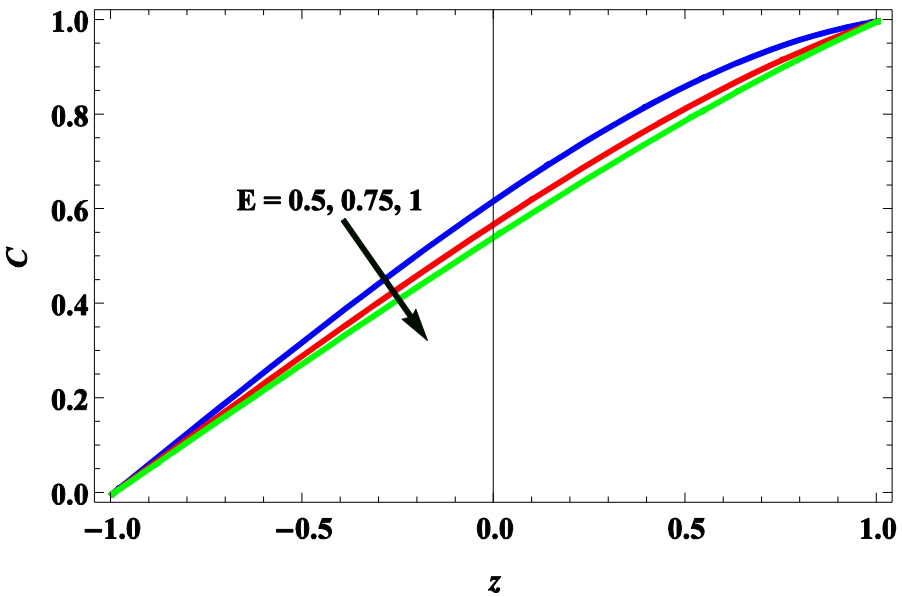

Fig. 13. Concentration profile for $E$.

Table 1 Skin friction coefficient at the non-magnetic and magnetized surfaces

\begin{tabular}{lcccccccc}
\hline$\gamma$ & $H e$ & $P m$ & $E$ & $k_{v}$ & $\tau_{x 1}$ & $\tau_{y 1}$ & $-\tau_{x 2}$ & $-\tau_{y 2}$ \\
\hline $\mathbf{0}$ & $\mathbf{0 . 5}$ & $\mathbf{0 . 7}$ & $\mathbf{0 . 5}$ & $\mathbf{0 . 2 5}$ & $\mathbf{0 . 6 2 0 1}$ & $\mathbf{1 . 5 7 4 4}$ & $\mathbf{7 . 8 3 8 5}$ & $\mathbf{3 . 7 6 7 4}$ \\
$\pi / 6$ & & & & & $\mathbf{0 . 7 6 9 8}$ & $\mathbf{1 . 4 8 3 8}$ & $\mathbf{8 . 2 4 0 7}$ & $\mathbf{3 . 5 6 2 0}$ \\
$\pi / 4$ & & & & & 0.9475 & 1.3762 & 8.7179 & 3.3184 \\
$\pi / 3$ & & & & & 1.1791 & 1.2361 & 9.3398 & 3.0009 \\
& $\mathbf{0 . 2 5}$ & & & & 1.2127 & 0.7785 & 8.6751 & 1.7994 \\
& $\mathbf{0 . 7 5}$ & & & & 0.3429 & 2.1395 & 7.5889 & 5.2318 \\
& & $\mathbf{0 . 5}$ & & & 0.9245 & 0.7255 & 7.8600 & 3.3039 \\
& & $\mathbf{0 . 9}$ & & & 0.8951 & 2.0505 & 8.3626 & 3.7223 \\
& & & $\mathbf{0 . 7 5}$ & & 0.6877 & 2.2777 & 6.4962 & 4.4040 \\
& & & $\mathbf{1}$ & & 0.8740 & 2.6354 & 5.3226 & 4.5769 \\
& & & & $\mathbf{0 . 1 5}$ & 0.1235 & 3.2428 & 8.7801 & 5.1386 \\
& & & & $\mathbf{0 . 3 5}$ & 1.4646 & 0.7431 & 7.9789 & 2.8647 \\
\hline & & & & & & & &
\end{tabular}

Table 2 Heat and mass transfer rates at the non-magnetic and magnetized surfaces

\begin{tabular}{lllll}
\hline$E$ & $N u_{1}$ & $N u_{2}$ & $S h_{1}$ & $S h_{2}$ \\
\hline $\mathbf{0 . 5}$ & 0.2964 & 0.5666 & 0.6545 & 0.1268 \\
$\mathbf{0 . 7 5}$ & 0.2818 & 0.8161 & 0.5930 & 0.2826 \\
$\mathbf{1}$ & 0.2629 & 0.9562 & 0.5575 & 0.3655 \\
\hline
\end{tabular}

\section{Conclusions}

This paper deals with the analytical study of Hall and induced magnetic field effects on convective flow of viscoelastic fluid within an inclined channel with periodic surface conditions. The impacts of various system parameters to the flow behaviour are analyzed 
Name of First Author et al.

International Journal of Thermofluid Science and Technology (2020), Volume 7, Issue 4, Paper No. 070402

with assistance of graphs and tables. The novel results have relevance in the study of fluid engineering and bio-chemical processes. Some important observations are outlined below:

- The angle of inclination leads to a decrement in the velocity because on raising the angle of inclination buoyancy forces fall down. It brings increment in the induced magnetic field in the direction perpendicular to the main flow.

- Hall current has reducing tendency on the main flow while it has growing tendency on the flow perpendicular to the main flow. It brings decrement in the induced magnetic field along the main flow.

- Viscoelastic parameter tends to grow the main flow while it reduces the flow perpendicular to the main flow. Induced magnetic field along the main flow grows on growing the viscoelastic parameter.

\section{Acknowledgement}

Authors are thankful to the reviewer(s) for their valuable comments and suggestions which helped us to improve the quality of the work.

\section{References}

[1] U. Mishra and G. Singh, MHD mixed convection and mass transfer due to unsteady stretching sheet, Heat Trans. Asian Res., 43 (2014) 447-458.

[2] M. M. Rashidi, M. Ali, N. Freidoonimehr, B. Rostami and M. Anwar Hossain, Mixed convective heat transfer for MHD viscoelastic fluid flow over a porous wedge with thermal radiation, Adv. Mech. Eng., 2014 (2014) ArticleID 735939, 10 pages.

[3] M. Sajid, B. Ahmed and Z. Abbs, Steady mixed convection stagnation point flow of MHD Oldroyd-B fluid over a stretching sheet, J. Egyptian Math. Soc., 23 (2015) 440-444.

[4] M. M. Nejad, K. Javaherdeh and M. Moslemi, MHD mixed convection flow of power law non-Newtonian fluids over an isothermal vertical wavy plate, J. Mag. Mag. Mat., 389 (2015) 66-72.

[5] G. S. Seth and J. K. Singh, Mixed convection hydromagnetic flow in a rotating channel with Hall and wall conductance effects, Applied Mathematical Modelling, 40 (2016) 2783-2803.

[6] L. Ramamohan Reddy, M. C. Raju, G. S. S. Raju and N. A. Reddy, Thermal diffusion and rotational effects on magnetohydrodynamic mixed convection flow of heat absorbing/generating viscoelastic fluid through a porous channel, Frontiers in Heat and Mass Transfer., 7(1) (2016) 20.

[7] I. Ahmad, A. Abbasi, W. Abbasi and W. Farooq, Mixed convective peristaltic flow of an Oldroyd 4-constant fluid in a planner channel, Int. J. Thermofluid Sci. Tech., 6(3) (2019) 19060302.

[8] J. K. Singh, S. G. Begum and G. S. Seth, Influenced of Hall current and wall conductivity on hydromagnetic mixed convective flow in a rotating Darcian channel, Physics of Fluids, 30 (2018) 113602.

[9] J. K. Singh, G. S. Seth, Naveen Joshi and C. T. Srinivasa, Mixed convection flow of a viscoelastic fluid through a vertical porous channel influenced by a moving magnetic field with Hall and ion-slip currents, rotation, heat radiation and chemical reaction, Bulgarian Chemical Communications, 52 (2020) 147-158. 
Name of First Author et al.

International Journal of Thermofluid Science and Technology (2020), Volume 7, Issue 4, Paper No. 070402

[10] J. K. Singh, G. S. Seth, S. Vishwanath and P. Rohidas, Steady MHD mixed convection flow of a viscoelastic fluid over a magnetized convectively heated vertical surface with Hall current, rotation and induced magnetic field effects, Heat transfer, (2020). https://doi.org/10.1002/htj.21831

[11] A. A. Opanuga, S. O. Adesanya, H. I. Okagbue and O. O. Agboola, Impact of Hall current on the entropy generation of radiative MHD mixed convection Casson fluid, Int. J. Appl. Comput. Math., 6(44) (2020). https://doi.org/10.1007/s40819-020-0790-0.

[12] G. S. Seth and S. Sarkar, Hydromagnetic natural convection flow with induced magnetic field and nth order chemical reaction of a heat absorbing fluid past an impulsively moving vertical plate with ramped temperature, Bulg. Chem. Com., 47 (2015) 66-79.

[13] Sarveshanand and A. K. Singh, Magnetohydrodynamic free convection between vertical parallel porous plates in the presence of induced magnetic field, Springer Plus, 4 (2015) 333. https://doi.org/10.1186/s40064-015-1097-1

[14] D. Sarma and K. K. Pandit, Effects of thermal radiation and chemical reaction on steady MHD mixed convective flow over a vertical porous plate with induced magnetic field, Int. J. Fluid Mechanics Research, 42(4) (2015) 315-333.

[15] O. Ojjela, A. Raju and P. K. Kambhatla, Influence of thermophoresis and induced magnetic field on chemically reacting mixed convective flow of Jeffrey fluid between porous parallel plates, J. Molecular Liquids, 232 (2017) 195-206.

[16] Basant K. Jha and Babatunde Aina, Interplay of non-conducting and conducting walls on magnetohydrodynamic(MHD) natural convection flow in vertical micro-channel in the presence of induced magnetic field, Propulsion and power Research, 7 (4) (2018) 296-307.

[17] Dileep Kumar, A. K. Singh and Devendra Kumar, Effects of Hall current on the magnetohydrodynamic free convective flow between vertical walls with induced magnetic field, Eur. Phys. J. Plus, 133 (2018) 207. https://doi.org/10.1140/epjp/i2018-12012-4

[18] B. Kumar, G. S. Seth, R. Nandkeolyar and A. J. Chamkha, Outlining the impact of induced magnetic field and thermal radiation on magneto-convection flow of dissipative fluid, Int. J. Thermal Sci., 149 (2019) 106101.

[19] S. Das, B. Tarafdar, R. N. Jana and O. D. Makinde, Magnetic Ferro-Nanofluid fluid flow in a rotating channel containing Darcian porous medium considering induced magnetic field and Hall currents, Special Topics and Reviews in Porous Media: an Int. J., 10(4) (2019) 357-383.

[20] T. Hayat, W. A. Khan, S. Z. Abbas, S. Nadeem and S. Ahmad, Impacts of induced magnetic field on second-grade nanofluid flow past a convectively heated stretching sheet, Applied Nanoscience, 10 (2020) 3001-3009. https://doi.org/10.1007/s13204-019-01215-X

[21] M. Raza, R. Ellahi, Sadiq M. sait, M. M. Sarafraz, M. S. Shadloo and Ifraheem Waheed, Enhancement of heat transfer in peristaltic flow in a permeable channel under induced magnetic field using different CNT's, J. Thermal Analysis and Calorimetry, 140 (2020) 12771291.

[22] J. K. Singh, G. S. Seth and P. Rohidas, Impacts of time varying wall temperature and concentration on MHD free convective flow of a rotating fluid due to moving free-stream with Hall and ion-slip currents, Int. J. Thermofluid Sci. Tech., 6(3) (2019) 19060301.

[23] G. Dharmaiah, O. D. Makinde and K. S. Balamurugan, Perturbation analysis of thermophoresis, Hall current and heat source on flow dissipative aligned convective flow about an inclined plate. Int. J. Thermofluid Sci. Tech., 7(1) (2020) 20070103. 
Name of First Author et al.

International Journal of Thermofluid Science and Technology (2020), Volume 7, Issue 4, Paper No. 070402

[24] S. Nandi and B. Kumnhakar, Unsteady MHD free convective flow past a permeable vertical plate with periodic movement and slippage in the presence of Hall current and rotation, Thermal Science and Engineering Progress, 19 (2020) 100561.

[25] M. K. Nayak, G. C. Dash and L. P. Singh, Heat and mass transfer effects on MHD viscoelastic fluid over a stretching sheet through porous medium in presence of chemical reaction, Propulsion and Power Research, 5 (2016) 70-80.

[26] K. Ramesh and M. Devakar, Effect of Heat transfer on the peristaltic flow of Walters B fluid in a vertical channel with an external magnetic field, J. Aerosp. Eng., 29 (2016) 04015050.

[27] S. Baag, S. R. Mishra, G. C. Dash and M. R. Acharya, Entropy generation analysis for viscoelastic MHD flow over a stretching sheet embedded in a porous medium, Ain Shams Eng. J., 8 (2017) 623-632.

[28] A. Majeed, A. Zeeshan, S. Z. Alamri and R. Ellahi, Heat transfer analysis in ferromagnetic viscoelastic fluid flow over a stretching sheet with suction, Neural Comp. Appl., 30 (2018) 1947-1955.

[29] J. K. Singh, N. Joshi and P. Rohidas, Unsteady MHD natural convective flow of a rotating Walter's-B fluid over an oscillating plate with fluctuating wall temperature and concentration, J. Mech., 34 (2018) 519-532.

[30] J. K. Singh, G. S. Seth and S. G. Begum, Unsteady MHD natural convection flow of a viscoelastic fluid over an infinite vertical porous plate due to oscillating free-stream, Multidiscipline Modeling in Materials and Structures, 14 (2018) 236-260.

[31] S. Dorjee and U. J. Das, Unsteady MHD flow of viscoelastic fluid through a porous medium in a vertical porous channel, Emerging Technologies in Data Mining and Information Security, 755 (2019) 341-354.

[32] X. Chen, W. Yang, X. Zhang and F. Liu, Unsteady boundary layer flow of viscoelastic MHD fluid with a double fractional Maxwell model, Applied Mathematics Letters, 95 (2019) 143 149.

[33] F. Ali, M. Bilal, M. Gohar, I. Khan, N. A. Sheikh and K. S. Nisar, A Report on fluctuating free convection flow of heat absorbing viscoelastic Dusty fluid past in a horizontal channel with MHD effect, Scientific Reports, 10 (2020) 8523.

[34] S. Mishra, A. Misra and M. K. Nayak, Flow and heat transfer of Oldroyd-B nanofluid with relaxation-retardation viscous dissipation and hyperbolic boundary conditions, Int. J. Thermofluid Sci. Tech., 7(1) (2020) 20070104.

[35] M. Veera Krishna and A. J. Chamkha, Hall and ion slip effects on MHD rotating flow of elastico-viscous fluid through porous medium, Int. Comm. Heat and Mass Transfer, 113 (2020) 104494.

[36] J. K. Singh, G. S. Seth, S. G. Begum and S. Vishwanath, Hydromagnetic free convection flow of Walters' B fluid over a vertical surface with time varying surface condition, World Journal of Engineering, 17 (2) (2020) 295-307.

[37] D. S. Cimpean and I. Pop, Fully developed mixed convection flow of a nanofluid through an inclined channel filled with a porous medium, Int. J. Heat and Mass Transfer, 55 (2012) 907914.

[38] A. V. Ramanakumari and G. Radhakrishnamacharya, Effect of slip and magnetic field on peristaltic flow in an inclined channel with wall effects, Int. J. Biomathematics, 5(6) (2012) 1250015 . 
Name of First Author et al.

International Journal of Thermofluid Science and Technology (2020), Volume 7, Issue 4, Paper No. 070402

[39] D. Srinivasacharya and K. Hima Bindu, Entropy generation in a micropolar fluid flow through an inclined channel with slip and convective boundary conditions, Entropy, 91 (2015) $72-83$.

[40] T. Hayat, M. Shafique, A. Tanveer and A. Alsaedi, Magnetohydrodynamic effects on peristaltic flow of hyperbolic tangent nanofluid with slip conditions and Joule heating in an inclined channel, Int. J. Heat and Mass Transfer, 102 (2016) 54-63.

[41] H. Mondal, D. pal, S. Chatterjee and P. Sibanda, Thermophoresis and Soret-Dufour on MHD mixed convection mass transfer over an inclined plate with non-uniform heat source/sink and chemical reaction, Ain Shams Eng. J., 9 (4) (2018) 2111-2121.

[42] Ramesh Katta and M. Dewakar, Influence of magnetohydrodynamics on peristaltic flow of a Walter's B-fluid in an inclined asymmetric channel with heat transfer, World J. Eng., 15 (4) (2018) 450-467.

[43] D. Talukdar, Chung-Gang $\mathrm{Li}$ and M. Tsubokura, Numerical investigation of laminar compressible natural convection flow in asymmetrically and isothermally heated open-ended inclined channel, Int. J. Heat and Mass transfer, 130 (2019) 83-97.

[44] F. Garcia, C. Trevino, J. Lizardi and L. Martinez-suastegui, Numerical study of buoyancy and inclination effects on transient mixed convection in a channel with two facing cavities with discrete heating, Int. J. Mechanical Sciences, 155 (2019) 295-314.

[45] R. S. Raju, G. J. Reddy, M. Anil Kumar and R. S. R. Gorla, Jeffrey fluid impact on MHD free convective flow past a vertically inclination plate with transfer effects: EFGM solutions, Int. J. Fluid Mech. Res., 46(3) (2019) 239-260.

[46] T. Sharma, P. Sharma and N. Kumar, Analysis of Entropy generation due to MHD natural convective flow in an inclined channel in the presence of magnetic field and heat source effects, BioNanoScience, 9 (2019) 660-671.

[47] A. T. Ngiangia and F. L. Jim-George, Effects of electro-thermal conductivity on MHD free convection flow in an inclined porous channel, J. Information and Optimization Sciences, 40(6) (2019) 1265-1279.

[48] S. Noreen, T. Kausar, D. Tripathi, Quarat ul Ain and D. C. Lu, Heat transfer analysis on creeping flow carreau fluid driven by peristaltic pumping in an inclined asymmetric channel, Thermal Science and Engineering Progress, 17 (2020) 100486.

[49] M. A. Etzold, J. R. Landal and S. B. Dalziel, Three-dimensional advective-diffusive boundary layers in open channels with parallel and inclined walls, Int. J. Heat and Mass Transfer, 153 (2020) 119504. https://doi.org/10.17863/CAM.49368

[50] J. K. Singh and S. Vishwanath, Hall and ion-slip effects on MHD free convective flow of a viscoelastic fluid through porous regime in an inclined channel with moving magnetic field, Kragujevac J. Sci., 42 (2020) 5-18.

\section{Appendix}

$$
\begin{aligned}
& r_{0}=\sqrt{S}, r_{1,2}=\sqrt{S \pm(i \omega \operatorname{Pr} / E)}, s_{0}=\sqrt{R c}, s_{1,2}=\sqrt{R c \pm(i \omega S c / E)}, \\
& E_{1}=\left(\left(E / k_{1}\right)+2 i\right), E_{2}=E_{1}-E a_{0}^{2}, \\
& a_{0}=\sqrt{E^{-1}\left\{\left(\left(E / k_{1}\right)+\left(2 \alpha_{m}^{2} /\left(1+H e^{2}\right)\right)\right)+2 i\left(1+\left(H_{e} \alpha_{m}^{2} /\left(1+H e^{2}\right)\right)\right)\right\}}
\end{aligned}
$$


Name of First Author et al.

International Journal of Thermofluid Science and Technology (2020), Volume 7, Issue 4, Paper No. 070402

$$
\begin{aligned}
& z_{11,21}=\left\{\left(\mp \frac{\omega P m H e}{E\left(1+H e^{2}\right)}+\frac{\left(E^{2} / k_{1}\right)+\omega k_{v}(\omega \pm 2)}{\left(E^{2}+\omega^{2} k_{v}^{2}\right)}+\frac{2 \alpha_{m}^{2}\left(E \pm \omega k_{v} H e\right)}{\left(E^{2}+\omega^{2} k_{v}^{2}\right)\left(1+H e^{2}\right)}\right)\right. \\
& \left.+i\left( \pm \frac{\omega P m}{E\left(1+H e^{2}\right)} \pm \frac{E\left((\omega \pm 2)-\left(\omega k_{v} / k_{1}\right)\right)}{\left(E^{2}+\omega^{2} k_{v}^{2}\right)}+\frac{2 \alpha_{m}^{2}\left(E H_{e} \mp \omega k_{v}\right)}{\left(E^{2}+\omega^{2} k_{v}^{2}\right)\left(1+H e^{2}\right)}\right)\right\}, \\
& z_{12,22}=\left\{\left(\frac{-\omega(\omega \pm 2) P m\left(E \pm \omega k_{v} H e^{2}\right) \mp\left(\omega P m E / k_{1}\right)\left(E H e \mp \omega k_{v}\right)}{E\left(E^{2}+\omega^{2} k_{v}^{2}\right)\left(1+H e^{2}\right)}\right)\right. \\
& \left.+i\left(\frac{ \pm\left(\omega P m E / k_{1}\right)\left(E \pm \omega k_{v} H e\right)-\omega(\omega \pm 2) P m\left(E H e \mp \omega k_{v}\right)}{E\left(E^{2}+\omega^{2} k_{v}^{2}\right)\left(1+H e^{2}\right)}\right)\right\}, \\
& z_{13.23}=\left(\frac{\mp \omega P m\left(E H e \mp \omega k_{v}\right) \pm i \omega P m\left(E \pm \omega k_{v} H e\right)}{E\left(E^{2}+\omega^{2} k_{v}^{2}\right)\left(1+H e^{2}\right)}\right), z_{14,24}=\frac{E\left(E \mp i \omega k_{v}\right)}{\left(E+\omega^{2} k_{v}^{2}\right)} \text {, } \\
& z_{15,25}=\left(\frac{E\left(E H e \mp \omega k_{v}\right)+i E\left(E \pm \omega k_{v} H e\right)}{\omega P m}\right), z_{7}=\frac{\left(E^{2} H e+i E^{2}\right)}{\omega P m}, \\
& z_{16,26}=\left(\frac{E\left(\left(E H e / k_{1}\right) \mp(\omega \pm 2)\right)+i E\left(\left(E / k_{1}\right) \pm H e(\omega \pm 2)+2 \alpha_{m}^{2}\right)}{\omega P m}\right), \\
& a_{11,12}=\left(1 / 2^{1 / 2}\right)\left\{\left(z_{11}-4 z_{12}\right)^{1 / 2} \pm z_{11}\right\}^{1 / 2}, a_{21,22}=\left(1 / 2^{1 / 2}\right)\left\{\left(z_{21}-4 z_{22}\right)^{1 / 2} \pm z_{21}\right\}^{1 / 2} \\
& a_{i 1}^{\prime}=\left(a_{i 1}^{2} z_{i 5}-z_{i 6}\right) a_{i 1}, a_{i 2}^{\prime}=\left(a_{i 2}^{2} z_{i 5}-z_{i 6}\right) a_{i 2} \text {, } \\
& \left.r_{i 1}^{\prime}=\left(r_{i}^{2} z_{i 5}-z_{i 6}\right) r_{i}-\frac{z_{7} r_{i}\left(r_{i}^{4}-z_{i 1} r_{i}^{2}+z_{i 2}\right)}{\left(r_{i}^{2} z_{i 4}-z_{i 3}\right)}, \quad\right\} i=1,2 \\
& s_{i 1}^{\prime}=\left(s_{i}^{2} z_{i 5}-z_{i 6}\right) s_{i}-\frac{z_{7} s_{i}\left(s_{i}^{4}-z_{i 1} s_{i}^{2}+z_{i 2}\right)}{\left(s_{i 1}^{2} z_{i 4}-z_{i 3}\right)}, \\
& c_{01}=\left(E_{1}-\frac{E_{2} \sinh \left(a_{0}\right)}{a_{0} \cosh \left(a_{0}\right)}\right)^{-1}\left[R+\alpha_{m}^{2} E^{1 / 2}+\frac{1}{2} E_{2}\left\{\frac{1-\cosh \left(2 a_{0}\right)}{a_{0} \sinh \left(2 a_{0}\right)}\right.\right. \\
& \left.\left.+\frac{G r \cos \gamma}{\left(r_{0}^{2}-a_{0}^{2}\right)}\left(\frac{1-\cosh \left(2 a_{0}\right)}{a_{0} \sinh \left(2 a_{0}\right)}-\frac{1-\cosh \left(2 r_{0}\right)}{r_{0} \sinh \left(2 r_{0}\right)}\right)+\frac{G \operatorname{scos} \gamma}{\left(s_{0}^{2}-a_{0}^{2}\right)}\left(\frac{1-\cosh \left(2 a_{0}\right)}{a_{0} \sinh \left(2 a_{0}\right)}-\frac{1-\cosh \left(2 s_{0}\right)}{s_{0} \sinh \left(2 s_{0}\right)}\right)\right\}\right] \\
& c_{02}=\alpha_{m}^{2} E^{1 / 2}-\frac{1}{2} E_{2}\left\{\frac{1+\cosh \left(2 a_{0}\right)}{a_{0} \sinh \left(2 a_{0}\right)}+\frac{G r \cos \gamma}{\left(r_{0}^{2}-a_{0}^{2}\right)}\left(\frac{1+\cosh \left(2 a_{0}\right)}{a_{0} \sinh \left(2 a_{0}\right)}-\frac{1+\cosh \left(2 r_{0}\right)}{r_{0} \sinh \left(2 r_{0}\right)}\right)\right. \\
& \left.+\frac{G \cos \gamma}{\left(s_{0}^{2}-a_{0}^{2}\right)}\left(\frac{1+\cosh \left(2 a_{0}\right)}{a_{0} \sinh \left(2 a_{0}\right)}-\frac{1+\cosh \left(2 s_{0}\right)}{s_{0} \sinh \left(2 s_{0}\right)}\right)\right\}
\end{aligned}
$$




$$
\begin{aligned}
c_{11,21}= & \frac{\cosh \left(a_{11,21}\right)}{\left(a_{12,22}^{\prime} \sinh \left(a_{12,22}\right) \cosh \left(a_{11,21}\right)-a_{11,21}^{\prime} \sinh \left(a_{11,21}\right) \cosh \left(a_{12,22}\right)\right)} \\
\times & {\left[ \pm \alpha_{m}^{2} E^{1 / 2}+\frac{z_{13,23} R}{E z_{12,22}} \frac{a_{11,21}^{\prime} \sinh \left(a_{11,21}\right)}{\cosh \left(a_{11,21}\right)}+\frac{1}{2}\left\{\frac{a_{11,21}^{\prime}\left(1-\cosh \left(2 a_{11,21}\right)\right)}{\sinh \left(2 a_{11,21}\right)}\right.\right.} \\
+ & \frac{G r \cos \gamma\left(r_{1,2}^{2} z_{14,24}-z_{13,23}\right)}{\left(r_{1,2}^{4}-z_{11,21} r_{1,2}^{2}+z_{12,22}\right)}\left(\frac{a_{11,21}^{\prime}\left(1-\cosh \left(2 a_{11,21}\right)\right)}{\sinh \left(2 a_{11,21}\right)}-\frac{r_{11,21}^{\prime}\left(1-\cosh \left(2 r_{1,2}\right)\right)}{\sinh \left(2 r_{1,2}\right)}\right) \\
+ & \left.\left.\frac{G \operatorname{scos} \gamma\left(s_{1,2}^{2} z_{14,24}-z_{13,23}\right)}{\left(s_{1,2}^{4}-z_{11,21} s_{1,2}^{2}+z_{12,22}\right)}\left(\frac{a_{11,21}^{\prime}\left(1-\cosh \left(2 a_{11,21}\right)\right)}{\sinh \left(2 a_{11,21}\right)}-\frac{s_{11,21}^{\prime}\left(1-\cosh \left(2 s_{1,2}\right)\right)}{\sinh \left(2 s_{1,2}\right)}\right)\right\}\right] \\
c_{12,22}= & \frac{\sinh \left(a_{11,21}\right)}{\left(a_{12,22} \cosh \left(a_{12,22}\right) \sinh \left(a_{11,21}\right)-a_{11,21}^{\prime} \cosh \left(a_{11,21}\right) \sinh \left(a_{12,22}\right)\right)} \\
& \times\left[ \pm \alpha_{m}^{2} E^{1 / 2}-\frac{1}{2}\left\{\frac{a_{11,21}^{\prime}\left(1+\cosh \left(2 a_{11,21}\right)\right)}{\sinh \left(2 a_{11,21}\right)}\right.\right. \\
& +\frac{G r c o s \gamma\left(r_{1,2}^{2} z_{14,24}-z_{13,23}\right)}{\left(r_{1,2}^{4}-z_{11,21} r_{1,2}^{2}+z_{12,22}\right)}\left(\frac{a_{11,21}^{\prime}\left(1+\cosh \left(2 a_{11,21}\right)\right)}{\sinh \left(2 a_{11,21}\right)}-\frac{r_{11,21}^{\prime}\left(1+\cosh \left(2 r_{1,2}\right)\right)}{\sinh \left(2 r_{1,2}\right)}\right) \\
& \left.+\frac{G \operatorname{sos} \gamma\left(s_{1,2}^{2} z_{14,24}-z_{13,23}\right)}{\left(s_{1,2}^{4}-z_{11,21} s_{1,2}^{2}+z_{12,22}\right)}\left(\frac{a_{11,21}^{\prime}\left(1+\cosh \left(2 a_{11,21}\right)\right)}{\sinh \left(2 a_{11,21}\right)}-\frac{s_{11,21}^{\prime}\left(1+\cosh \left(2 s_{1,2}\right)\right)}{\sinh \left(2 s_{1,2}\right)}\right)\right\} .
\end{aligned}
$$

